\title{
Post-classification of nominally identical steel string guitars using bridge admittances
}

\author{
Hossein Mansour $^{\dagger 1}$, Vincent Fréour ${ }^{1}$, Charalampos Saitis ${ }^{1}$, and Gary P. Scavone ${ }^{1}$ \\ ${ }^{1}$ Computational Acoustic Modeling Laboratory, Centre for Interdisciplinary Research in \\ Music Media and Technology (CIRMMT), Schulich School of Music, McGill University, \\ 555 Sherbrooke Street West, Montréal, Québec H3A 1E3, Canada
}

November 29, 2014

†'hossein.mansour@mail.mcgill.ca 


\begin{abstract}
Dynamic and acoustical measurements were conducted on 18 nominally identical acoustic guitars coming off the same production line and post-classified by the manufacturer as either bassy (i.e., with a more prominent bass response), mid-even (i.e., well-rounded and sounding even from string to string) or treble (i.e., with a brighter sound that cuts through the band). One goal was to find features of the guitar admittances that could be used to automatically classify them according to these categories. A second goal was to investigate whether experienced guitarists agreed with the classifications provided by the manufacturer. Physical properties were investigated independently and in conjunction with perceptual assessments by musicians collected during a classification task. Despite very low agreement across guitarists as well as between musicians and the manufacturer, results showed that the bassy guitars had a lower frequency for their breathing mode. This suggests a lower stiffness-to-weight ratio for the respective guitar bodies, which may be caused by small variations in the plate thickness or wood properties. The guitars characterized as treble in this study tended to have lower averaged mobility in the 600-2000 $\mathrm{Hz}$ range, which might suggest a weaker string-to-body coupling at those frequencies and/or a longer decay for higher partials, though these characteristics were not confirmed.
\end{abstract}

PACS numbers: 43.66.Jh, 43.75.Gh 


\section{Introduction}

This paper presents the results of a recent study investigating possible links between measurable characteristics of 18 nominally identical acoustic guitars and their perceived differences as classified by the manufacturer. The guitars were built in a modern factory with very precise control of geometrical conditions. But within a given guitar model, the manufacturer noted variations of sounding characteristics that were assumed to be due to natural variations in the wood used for their construction.

The study commenced with the manufacturer providing 18 guitars from the same production line (model Godin Seagull Maritime SWS) and post-classified by a single employee at the factory as either bassy (i.e., with a more prominent bass response), mid-even (i.e., well-rounded and sounding even from string to string) or treble (i.e., with a brighter sound that cuts through the band). One of the goals of the work was to identify measurable features of the guitars that could be used to automatically classify them. Another goal of the study was to investigate the level of agreement among guitar players in classifying the guitars into these categories. The measurement techniques used were chosen with an eye toward eventual implementation in a factory setting, where vibroacoustic isolation would likely be limited and the ease and repeatability of the measurements would be critical.

The measurements performed included input admittance at the bridge and long-term average spectra (LTAS) from live acoustical recordings. A few wire-breaking tests were conducted but a complete set of measurements across all guitars was not possible in the limited project time frame. Overall, results showed that guitars categorized as bassy by the manufacturer had a lower breathing mode frequency. The treble guitars demonstrated a lower averaged mobility in the frequency range of $600-2000 \mathrm{~Hz}$.

A perceptual experiment was conducted in which 13 experienced guitarists were asked to categorize the 18 guitars into the three above-mentioned categories. In general, there was very low agreement among the players in terms of the classifications. That said, there was more agreement on the bassy classifications than the other two categories.

This paper is organized as follows: Section 2 provides background on measurement and analysis results previously described in the literature. Section 3 reports the results of the physical measurements conducted in this study and analyses related to the guitar categorizations. Sec- 
tion 4 describes the perceptual experiment conducted and its results. Finally, the overall results are considered in Section 5, with thoughts on possible future work that could be performed to help clarify the results.

\section{Background}

The energy of a vibrating string is primarily transmitted to the soundboard of the guitar through the bridge. The mechanical input admittance at the bridge (or bridge mobility) is defined in the frequency domain and in a given direction as the ratio of the velocity to an input force driving the structure at a given location. It provides a description of the ability of a point of the sound board to be displaced as a function of frequency. Therefore, this quantity relates to the ability of the sound board to move and to radiate acoustic energy, while it also describes the amount of energy absorbed from the string, which ultimately affects the decay rate of string vibration. Consequently, measurements of the bridge mobility are likely to provide relevant information with regards to the vibrational characteristics of an instrument.

The top plate of the guitar constitutes the primary radiating element of the instrument and thus, it plays a very important role in the characteristics of the radiated sound. The analysis of the modal shapes of the lowest frequency guitar modes reveals that the higher mobility of the top plate occurs on its lower bout, the upper bout presenting less mobility [1]. In the amplitude curve of the frequency response of the instrument measured at the bridge of the instrument, these low-frequency modes can be easily identified and are commonly designated in reference to their physical origins.

Different discrete models have been proposed to represent the low frequency and weakly damped modal response of the guitar body. Christensen [2] presented a simple two-degree of freedom model that allows a description of the coupling between the soundbox and the Helmholtz resonance and subsequently, a three-degree of freedom model including an additional mass to represent the back plate of the guitar $[3,4]$. That model identifies three coupled modes which are the result of the strong interaction between the first bending modes of the top and back plates as well as the Helmholtz resonance of the cavity. The first of the three is the breathing mode of the soundbox (hereafter $\left.\mathrm{T}(1,1)_{1}\right)$, in which the top and back plate move out-of-phase. This leads to the expansion and contraction of the soundbox resulting in the air 
being sucked in or pushed out through the sound hole respectively. In the second mode the two plates move in-phase while the ribs move in the opposite direction. Changing the boundary condition around the ribs will thus affect the frequency of this mode fairly strongly (as much as $30 \mathrm{~Hz}[5]$ ). The third mode (hereafter $\mathrm{T}(1,1)_{2}$ or anti-breathing mode) is the same as the first, but instead the air is being pushed out when the soundbox expands and vice versa. Typically there is an anti-resonance between the first and the second modes at the frequency where the pure Helmholtz mode would fall if the soundbox was rigid. Among the three, the first and the third modes involve a change in the volume of the cavity and thus radiate well, while the second mode does not radiate well. More recently, Popp proposed a four-degree of freedom model [6]. By including a fourth oscillator representing the motion of guitar sides and neck, this model allows a more precise exploration of the influence of rib mass on the location of the low-frequency resonances. There are a few other modes below $400 \mathrm{~Hz}$ that involve a coupling between the top and back plates; above that frequency, however, top plate, back plate, and air cavity behave relatively independently.

For most structures the bandwidth of individual modes increases roughly linearly with frequency, while the average spacing between adjacent modes remains almost constant. This results in an increasingly stronger interaction between close modes. At higher frequencies, the modes overlap to define the response of the system, which makes it more meaningful to think about statistical features rather than individual modes. In this regard, Modal Overlap Factor (MOF) is a useful criterion to find the strength of the interaction between close modes. MOF is the ratio of the bandwidth of individual resonance peaks to the average spacing of adjacent resonances and is expected to grow at higher frequencies [7]. Common MOF values for characterizing the different frequency ranges are MOF $<0.3$ for low frequencies, $0.3<\mathrm{MOF}<1$ for mid-frequencies and MOF $>1$ for high frequencies [8]. In the higher frequency range, quantities such as MOF, averaged admittance, average peak spacing, and typical peak-to-valley heights provide useful information that can potentially allow for the differentiation between two nearly identical instruments.

Recent approaches involve Statistical Energy Analysis (SEA) of the frequency response over a broader frequency range [9] and high-resolution modal analysis based on the modeling of the impulse response of a structure as a sum of complex damped sinusoids [8]. Using the latter approach, Elie et al. proposed a few macro dynamic features for discriminating between different 
classes of classical guitars. Although the low-quality ("industrial") guitars were carved out of a different material (plywood) than the high-quality ("hand-made") instruments (made with spruce or red cedar), the results illustrate the potential of that approach to associate global physical quantities of a guitar with construction details.

Long-term average spectra (LTAS) offers an alternative approach to the analysis of sound from complex sources such as musical instruments [10]. LTAS is the mean of successive shortterm spectra computed over the segments of a signal. This method reduces the noise in the estimated power spectra in exchange for reducing the frequency resolution [11], thereby highlighting longer term aspects of perceived sound quality. LTAS has been used extensively for studying perceived differences in speech and singing voices (e.g., [12]) but less often for quantifying instrumental sound quality $[10,13]$.

\section{Physical measurements}

\subsection{Method}

\subsubsection{Input admittance}

As mentioned in the previous section, the mechanical admittance measured at the bridge provides useful information regarding the ability of the sound board to be excited by the strings. We chose to perform measurement of the bridge admittance (or mobility) as well as the radiativity (transfer function between the input force at the bridge and the acoustic pressure radiated at a given point outside the instrument) on the set of guitars provided by the manufacturer. As shown in Figure 1 the guitar was held vertically by means of a wooden stand; the guitar was maintained by two rigid bars covered with foam holding the ribs just below the upper bout, at the level where the body of the instrument is the narrowest, without exerting too much pressure on the ribs. The strings were properly tuned and then damped using a clamp with foam fixed between the 9 th and 10th frets. The part of the strings above the nut was also damped using a piece of cardboard.

Energy was injected into the system at the bridge between the low E and A strings using an impact hammer (PCB Model 086E80) mounted on a pendulum allowing relatively repeatable impacts normal to the surface of the bridge. Mobility measurements require collocated excitation 


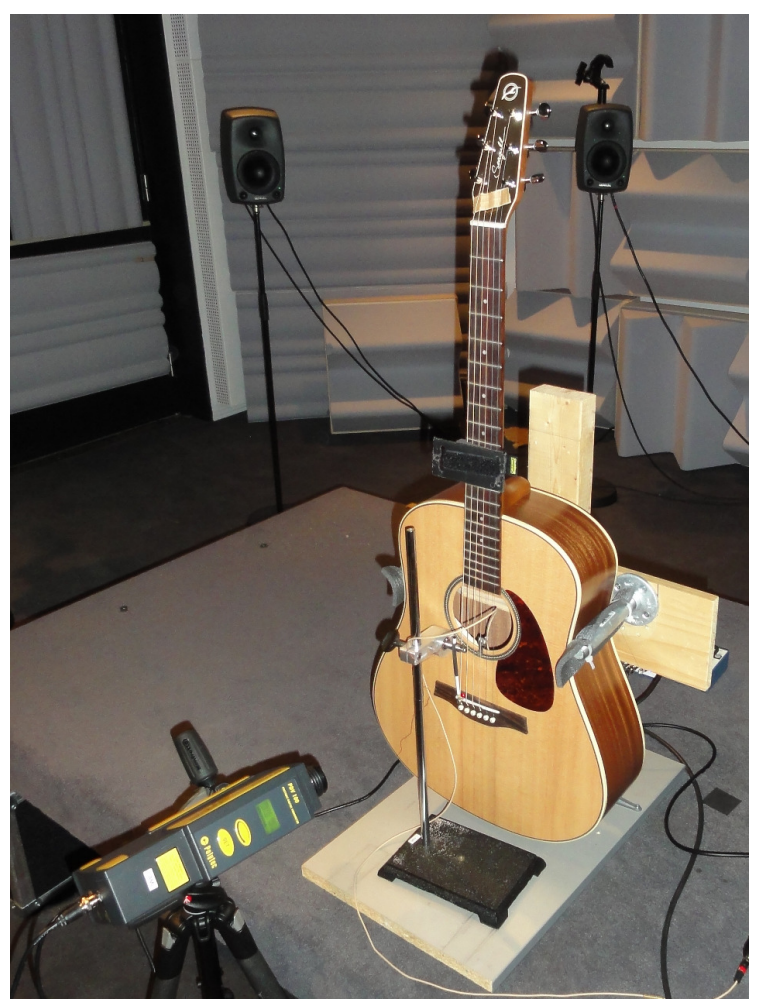

Figure 1: Experimental setup for input admittance and radiativity measurements.

and acquisition of the velocity. The velocity was hence measured as close as possible from the impact point between the E and A strings using a velocimeter (Polytec LDV-100) pointing normal to the surface of the bridge. The particular choice of bass side of the bridge was made due to the fact that a) some information about the symmetric and anti-symmetric modes of the body (with respect to the longitudinal axis of the instrument) might be lost if the measurements were made near the middle of the bridge, and b) the lowest string is the one that is most strongly coupled to the body, hence measuring the bridge admittance underneath that string can potentially provide the most relevant information about the body. A microphone (Brüel \& Kjær Type 4190-L-001) was placed one meter from the instrument, at the level of the neck to body junction, pointing towards the center of the sound hole. The measurements took place in a semi-anechoic room with dimensions of $5.4 \times 6.4 \times 3.6 \mathrm{~m}$. After appropriate pre-amplification of the acquired signals, acquisition was performed using a National Instruments data acquisition card running at $44.1 \mathrm{kHz}$ and a Matlab interface. Each measurement was of three-second duration after the beginning of the impact. For each guitar, final measurements were averaged over five repetitions. At each acquisition, the coherence was calculated and displayed to the 
operator, allowing any erroneous measurement to be discarded and repeated if necessary.

\subsubsection{Modal fitting}

Mode fitting was performed using an analysis method and algorithm developed by Woodhouse [9]. This method uses the Matlab function invfreqs allowing rational fraction polynomial approximation. It first involves modal extraction through pole-residue fitting. This first step is followed by an optimization procedure allowing selection of the best sets of complex and real residues by minimizing the mean of the modulus-squared deviation between measurement and reconstruction.

\subsubsection{Recordings and LTAS}

For the LTAS, all 18 guitars were recorded by a professional guitar player. The choice of musical input for the recorded sample sounds can have a significant influence on the LTAS obtained. On each of the guitars, we recorded a sequence of 8 strummed chords selected to excite a wide range of frequencies: $\mathrm{E}\left(\mathrm{E}_{2} \mathrm{~B}_{2} \mathrm{E}_{3} \mathrm{G}_{\sharp_{3}} \mathrm{~B}_{3} \mathrm{E}_{4}\right) \rightarrow \mathrm{F} \sharp \mathrm{m} 7\left(\mathrm{~F}_{2} \mathrm{E}_{3} \mathrm{~F}_{3} \mathrm{~A}_{3} \mathrm{C}_{4}\right) \rightarrow \operatorname{Emaj7}\left(\mathrm{E}_{2} \mathrm{~B}_{2}\right.$ $\left.\mathrm{D}_{3} \mathrm{G}_{3} \sharp_{3} \mathrm{~B}_{4}\right) \rightarrow \mathrm{A} / \mathrm{E}\left(\mathrm{E}_{2} \mathrm{~A}_{2} \mathrm{E}_{3} \mathrm{~A}_{3} \mathrm{C}_{4}\right) \rightarrow \mathrm{F} \sharp \mathrm{m} 7\left(\mathrm{~F}_{2} \mathrm{C}_{2} \sharp_{3} \mathrm{E}_{3} \mathrm{~A}_{3} \mathrm{E}_{4}\right) \rightarrow \operatorname{Emaj} 7\left(\mathrm{E}_{2} \mathrm{G}_{3} \mathrm{~B}_{3}\right.$ $\left.\mathrm{D}_{4} \mathrm{G}_{4}\right) \rightarrow \mathrm{A} / \mathrm{E}\left(\mathrm{E}_{3} \mathrm{~A}_{3} \mathrm{C}_{\sharp_{4}} \mathrm{E}_{4} \mathrm{~A}_{4}\right) \rightarrow \mathrm{E}\left(\mathrm{E}_{3} \mathrm{G}_{3} \mathrm{~B}_{3} \mathrm{E}_{4} \mathrm{G}_{4}\right)$. The musician was instructed to play mezzoforte at a tempo of 80 beats per minute.

The recordings took place in the same room as the admittance measurements. A 1/2-inch free-field microphone (Brüel \& Kjær Type 4190-L-001 with Type 2669-L preamplifier) with a sound quality conditioning amplifier (Brüel \& Kjær Type 2672) was used. The microphone was positioned $75 \mathrm{~cm}$ from the played guitar, facing directly at its top side, between the center of the sound hole and the fingerboard. The recorded phrases were 30 seconds long and saved in 16-bit, stereo $44.1 \mathrm{kHz}$ WAV format.

LTAS were obtained using the Matlab function pwelch, symmetric four-term BlackmanHarris windows, an 8192-point window size and 50\% overlap between the windows. Subsequently, the results were smoothed in the frequency domain using a $500 \mathrm{~Hz}$ sliding rectangular window. 


\subsection{Variation among the instruments}

We first evaluated the amount of variation among the 18 guitars. The precise manufacturing process in the production of these instruments made it almost impossible to see any appreciable geometrical difference among the individual instruments except a slight variation in the design of their necks (some were intentionally cut more flat and others more rounded). Therefore, the biggest uncertainty factor would be the variation in material properties. Although the instruments of our study were all made with spruce tops and mahogany backs and ribs, some variation of timbre is inevitable.

Figure 2 shows the averaged admittance over the pool of 18 instruments plus/minus one standard deviation. A sample instrument from the pool and an outlier are included for comparison. The outlier was a full-size acoustic guitar from the same manufacturer but of a different model. The standard deviation for all frequency points from $70 \mathrm{~Hz}$ to $6 \mathrm{kHz}$ averages to 2.75 $\mathrm{dB}$ when calculated for all 18 instruments. The level of variation is slightly higher if we look at the lower range of frequencies $(3.79 \mathrm{~dB}$ for the frequency range of $70 \mathrm{~Hz}$ to $1 \mathrm{kHz}$ ). As a reference, we calculated the standard deviation for 4 repeated measurements made on the same instrument, removed and put back on the fixture before each repetition. The standard deviation averaged to $0.54 \mathrm{~dB}$ for the range $70 \mathrm{~Hz}$ to $6 \mathrm{kHz}$, and $0.94 \mathrm{~dB}$ for the range $70 \mathrm{~Hz}$ to $600 \mathrm{~Hz}$. Note the different sample size when comparing these numbers with their equivalents reported earlier for the 18 instruments.

The same analysis can be applied to the instruments within the same perceptual categories (i.e., bassy, mid-even, and treble). Figure 3 shows the average admittance for the instruments of the different categories. The average standard deviations within the categories of bassy, mideven, and treble are respectively $2.63 \mathrm{~dB}, 2.36 \mathrm{~dB}$ and $2.15 \mathrm{~dB}$ for the frequency range of 70 $\mathrm{Hz}$ to $6 \mathrm{kHz}$; and $3.29 \mathrm{~dB}, 2.31 \mathrm{~dB}, 2.42 \mathrm{~dB}$ for the frequency range of $70 \mathrm{~Hz}$ to $1 \mathrm{kHz}$. The fact that the variation within each category is less than the variation among all the instruments lends some credibility to the classification made by the manufacturer. Another observation in Fig. 3 is that the mid-even and treble instruments follow almost the same trend below $600 \mathrm{~Hz}$, while bassy instruments are considerably distinct in the frequency range of $80 \mathrm{~Hz}$ to $100 \mathrm{~Hz}$ and again from $300 \mathrm{~Hz}$ to $350 \mathrm{~Hz}$, showing a shift of the main resonances towards lower frequencies. 


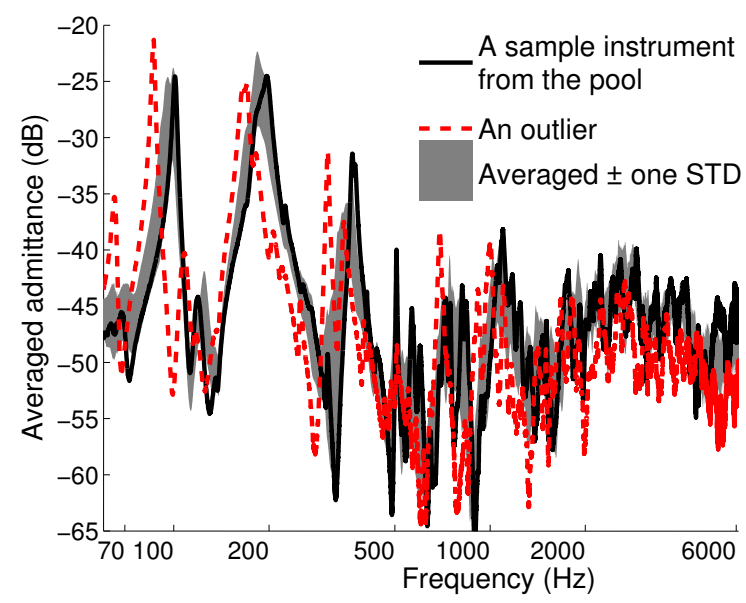

Figure 2: Gray shading shows the averaged admittance over the pool of 18 instruments plus/minus one standard deviation. The solid black line is a sample instrument from the pool and dashed line is an outlier. The $\mathrm{dB}$ reference is $1 \mathrm{~m} / \mathrm{sN}$.

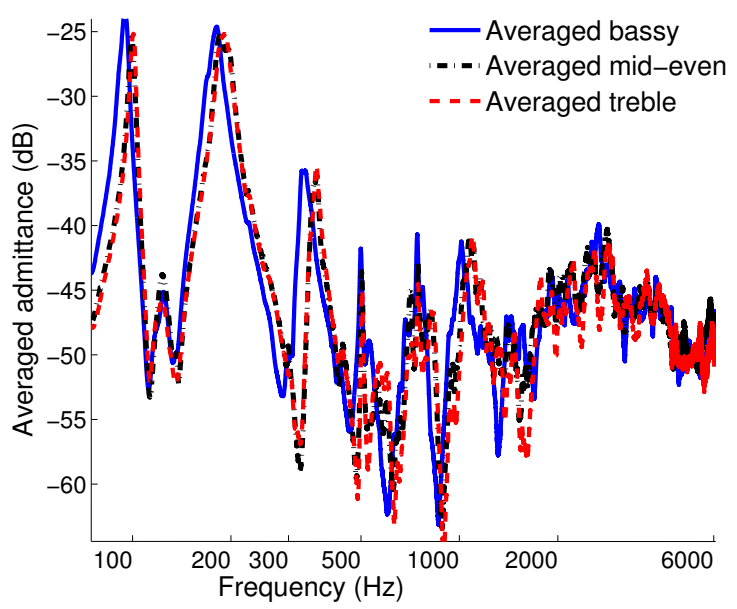

Figure 3: Averaged admittance over the bassy instruments (solid), mid-even instruments (dashdot), and treble instruments (dashed). The $\mathrm{dB}$ reference is $1 \mathrm{~m} / \mathrm{sN}$. 


\subsection{General properties of the acoustic guitars}

\subsubsection{Real-imaginary parts}

Figure 4 shows the real and imaginary parts of the averaged admittances, using an averaging bandwidth of $1000 \mathrm{~Hz}$. One can see that the acoustic guitar does not represent the properties of an infinite plate (i.e. positive real part with roughly constant magnitude, and imaginary part much smaller), at least not as well as a classical guitar does $[9,8]$. The magnitude of the imaginary part is smaller than the real part, but still large enough to be non-negligible. The real part stays positive over all frequencies, which is a property of any passive system. The average amplitude of the real part is smaller by almost a factor of two than the same measure for a typical classical guitar; it reaches the typical value for a classical guitar at around $3 \mathrm{kHz}$, and again drops at higher frequencies. A broad peak in the real part and a tendency in the imaginary part towards negative values creates a hill-like feature [14] at around $2800 \mathrm{~Hz}$ for the majority of the instruments, with a similar pattern that repeats less strongly at around 4 $\mathrm{kHz}$. These hills are quite unexpected as an acoustic guitar does not have a bridge like a violin, nor has it a feature like f-holes to create such local resonances. A possible explanation is that the cross-bracing of the steel string guitars, unlike the fan-bracing of most classical guitars, circumvents the bridge and forms an isolated area around it and this may contribute to such local resonances.

\subsubsection{Modal spacing and MOF}

It is useful to find a practical upper limit for the range of mode fitting (i.e. where the MOF reaches one) and to get a sense of the average spacing between the modes. Figure 5 shows the MOF and the average mode spacing for a sample instrument from the pool and the average plus/minus one standard deviation over all instruments. An averaging bandwidth of $500 \mathrm{~Hz}$ is used in this case to give a better precision at lower frequencies. The statistical features, including MOF, are calculated based on variance theory; for more details see equations (17) to (19) of [9]. As expected, the general trend of the overlap factor is rising, although it falls a bit between $1500 \mathrm{~Hz}$ and $2000 \mathrm{~Hz}$; it reaches the value of one at around $610 \mathrm{~Hz}$. The average mode spacing goes unrealistically high (as much as $150 \mathrm{~Hz}$ at around $200 \mathrm{~Hz}$ ) at lower frequencies. The mode spacing stays almost constant at around $20 \mathrm{~Hz}$ for the frequency range of 600-1200 Hz. 


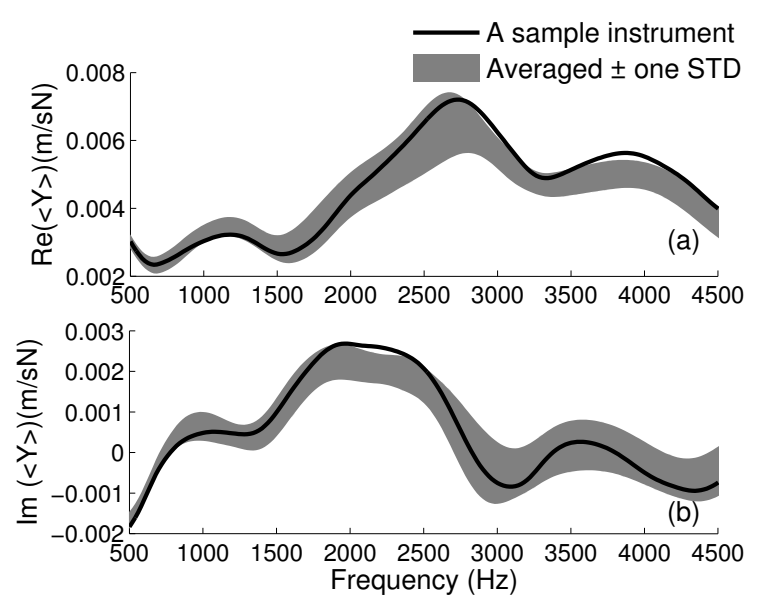

Figure 4: Band-averaged admittance for a sample instrument (solid), and averaged over all instruments of the pool plus/minus one standard deviation (shaded). Note that the vertical scales are different for the real and imaginary parts.

The mode spacing drops to around $8 \mathrm{~Hz}$ at higher frequencies and then stays almost unchanged.

\subsubsection{Mode fitting}

Using the method described in Sec. 2, a mode fitting process was performed on all 18 instruments of the pool up to $600 \mathrm{~Hz}$ [9]. The fitting program identified 20 to 24 modes for different instruments, amongst which a maximum of 16 modes were manually selected for each instrument. The criterion was to keep the stronger modes that were also identified for most of the instruments.

The general shape of the admittance follows the predictions of [6]: the first neck bending at around $65 \mathrm{~Hz}$, the $\mathrm{T}(1,1)_{1}$ or the breathing mode at around $100 \mathrm{~Hz}$, and $\mathrm{T}(1,1)_{2}$ or antibreathing mode at around $200 \mathrm{~Hz}$. Modes are categorized as $(m, n)$ where $m$ and $n$ are the number of "half-waves" across and along the plate, and T refers to the top plate. As pointed out by Popp [6], the in-phase motion of the top and back plates coincides in frequency with the anti-breathing mode at around $200 \mathrm{~Hz}$.

For our measurements, the guitars were held in a fixture with two arms that pressed against the sides of the ribs. This was intended to create a boundary condition similar to that when held by a player. Popp argues that a considerable amount of mass would be needed to immobilize the ribs, therefore our measurement condition is between the fixed and free-free cases, but perhaps more similar to the latter. After the measurements were performed, we observed a peak (or 


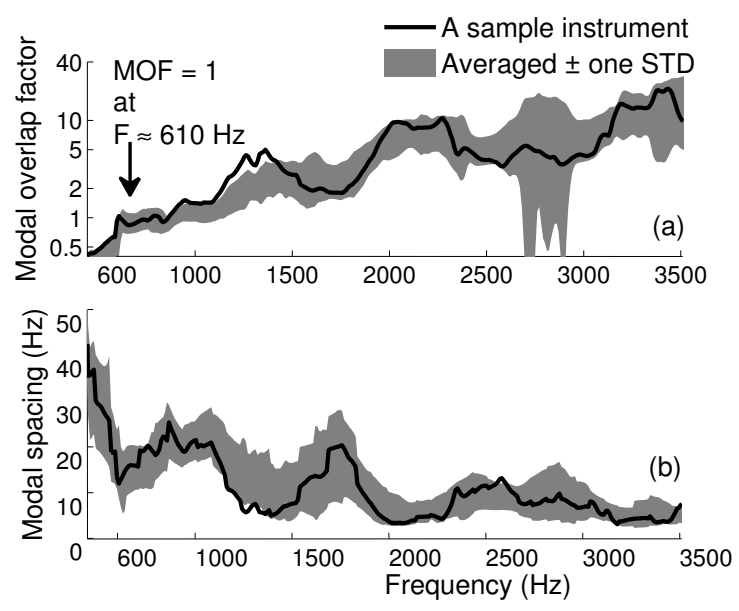

Figure 5: Modal overlap factor (top) and the average mode spacing (bottom) for a sample instrument from the pool (solid) and the average plus/minus one standard deviation over all instruments (shaded). An averaging bandwidth of $500 \mathrm{~Hz}$ is used.

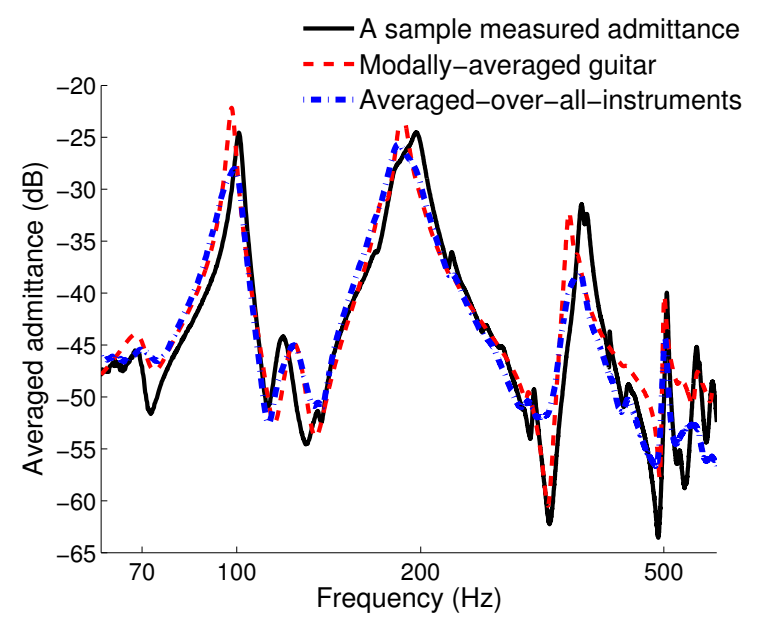

Figure 6: Raw averaged admittance over all instruments of the pool (dash-dot), reconstructed "modally-averaged" admittance (dashed, see text for details), and a sample admittance for comparison (solid). The $\mathrm{dB}$ reference is $1 \mathrm{~m} / \mathrm{sN}$. 
a double peak for some instruments) at around $125 \mathrm{~Hz}$ where one normally expects to see the anti-resonance representing the pure Helmholtz mode [2]. When we repeated the measurements on some of the guitars using a free-free boundary condition, we noticed that the anti-resonance appeared as expected. This suggests that either the peak is a property of the fixture rather than the instruments themselves, or it is the $(1,1)$ in-phase motion of the top and back plates shifted downward due to the mass of the fixture added to the ribs. The latter would require a $60 \mathrm{~Hz}$ downward shift of that frequency, which seems unlikely. No effort was made to repeat all the measurements with a free-free boundary condition, partly because the measurements were made before subsequent data analysis, and partly because free-free measurements are less practical and reproducible in a factory's production line (due to, for example, variations in accelerometer placement and potential damage to a new guitar surface finish when attaching and detaching an accelerometer).

The second neck bending mode was evident as a small peak at around $225 \mathrm{~Hz}$ for all instruments. There were two other small peaks at around $250 \mathrm{~Hz}$ and $290 \mathrm{~Hz}$ which were probably related to the torsion and lateral vibrations of the neck. The next important modes were $\mathrm{T}(2,1)$ and $\mathrm{T}(1,2)$. For Martin D-28 acoustic guitars these modes are around $100 \mathrm{~Hz}$ apart [5], hence relatively distinct; however, for the guitars of our study they both appear at around $350 \mathrm{~Hz}$ and sometimes switch places. We measured the admittance at the bass side of the bridge where $\mathrm{T}(2,1)$ has its antinode, therefore for each guitar the stronger mode in that region is associated to $\mathrm{T}(2,1)$. In addition to the Helmholtz mode, there are two other cavity modes in the frequency range of our study, which are $\mathrm{A} 1$ at $430 \mathrm{~Hz}$ and $\mathrm{A} 2$ at around $500 \mathrm{~Hz}$ [5]. Approximate mode shapes of the first four corpus modes (exclusive of local resonances, such as neck bending and neck twisting) for the guitars of our study are illustrated in Fig. 7; the frequencies are averaged and vary from one instrument to another.

The average frequency, amplitude, and Q-factors calculated for the 18 guitars were used to reconstruct the admittance of a "modally-averaged" guitar that represents the model that we studied. Figure 6 shows this "modally-averaged admittance" reconstructed up to $600 \mathrm{~Hz}$ (dashed line), compared with a sample measured admittance as well as the raw averaged admittance previously shown in Fig. 2. It can be seen that the peaks are excessively damped for the raw average, while the admittance of the "modally averaged" guitar shows a better resemblance to the sample measured admittance close to the resonances and anti-resonances. This modal 

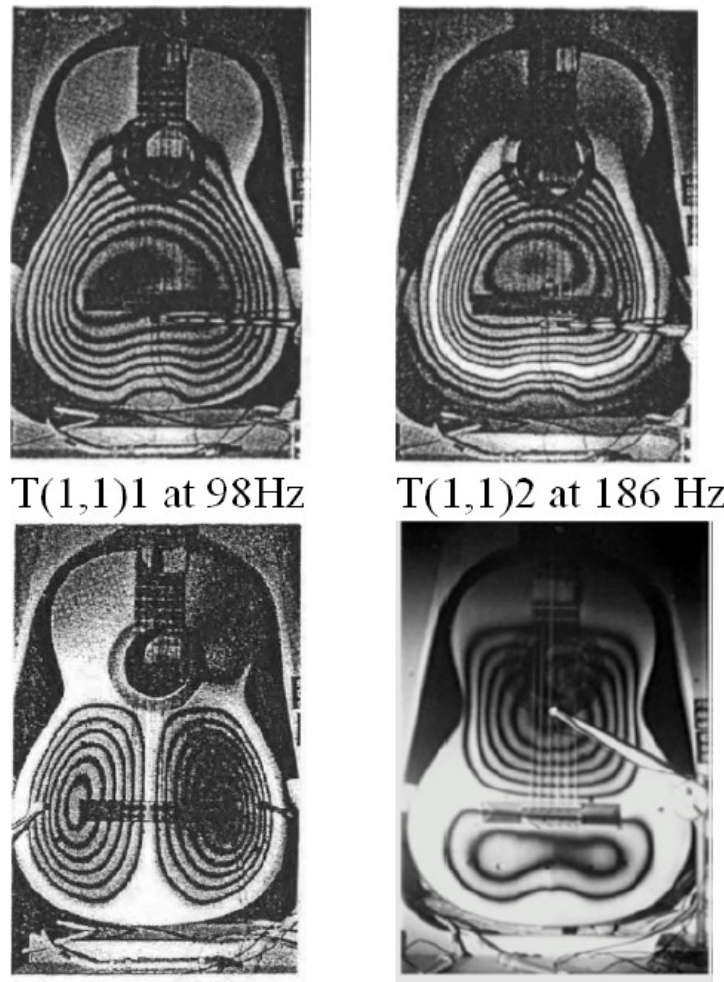

$\mathrm{T}(2,1)$ at $349 \mathrm{~Hz} \quad \mathrm{~T}(1,2) 1$ at $359 \mathrm{~Hz}$

Figure 7: The four first corpus mode shapes of an acoustic guitar, taken from [15]. 
average thus better represents the admittance of a guitar coming off this particular production line. It can be overlaid with the measured admittance of a new guitar to highlight its significant features.

If needed, the admittance of the "modally averaged" guitar can be reconstructed for extended frequencies either by simple averaging over different instruments or, more precisely, by using a "statistical fit" method as described in [16]. In short, a random number generator can give the frequency of the high-frequency modes with the correct density and spacing statistics, as well as damping factors, and modal masses with approximately correct statistical distribution. Such a statistical fit was not pursued in this study.

\subsubsection{LTAS}

Figure 8 shows the LTAS of a sample instrument from the pool (solid black line) as well as the average over all instruments plus/minus one standard deviation (shaded). The first few dominant modes identified in an admittance have demonstrated themselves in the form of broad peaks in the lower frequencies of the LTAS below about $400 \mathrm{~Hz}$. Some instruments such as the depicted sample show a peak at around $2.5 \mathrm{kHz}$, which may correspond to the hill-like feature earlier identified in band-averaged mobility curves (see Fig. 4). Beyond $300 \mathrm{~Hz}$, the LTAS drops almost linearly with the average slope of $16 \mathrm{~dB}$ /octave. The average standard deviation for the frequency range of $70 \mathrm{~Hz}$ to $6 \mathrm{kHz}$ for all instruments is $2.56 \mathrm{~dB}$, which indicates the player was able to play consistently on all instruments. It is worth mentioning that this number is not directly comparable to the averaged standard deviation for the measured admittance as the LTAS is significantly smoothed in the frequency domain in comparison.

\subsection{Investigating different features for classification}

\subsubsection{Modal properties}

Averaged value and standard deviation of frequency, amplitude, and Q factor of the first four corpus modes are calculated for the three perceptual classes and compared in Fig. 9. In all three figures the values are normalized to the average of the same modal value taken over all instruments and the error bars show one standard deviation on each side. In case of a missing mode for one instrument, its frequency and $\mathrm{Q}$ factor were eliminated from the calculation of 


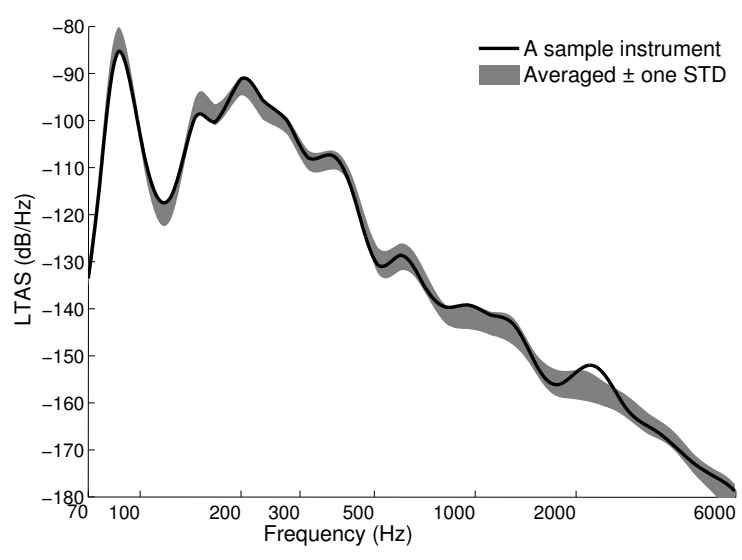

Figure 8: Long-term average spectra for a sample instrument from the pool (solid line) and the average plus/minus one standard deviation over all instruments (shaded).

the average and standard deviation, but the amplitude was considered to be zero (as that mode was negligible or non-existent). The admittance was not compensated for added damping from strings [9], partly because the exact same strings were used on all instruments, hence the comparison was fair, and partly because the exact amount of damping added by the strings is unknown, and the assumption of perfect damping at the strings may result in over-compensation of damping [9].

As was also evident from Fig. 3, Fig. 9a shows that the corpus modes of the bassy instruments occur at significantly lower frequencies than the mid-even or treble ones. Another feature that distinguishes bassy instruments from the mid-even or treble ones can be seen in Fig. 9b: the amplitude of the first corpus mode $\mathrm{T}(1,1)_{1}$ is higher for the bassy instruments. The $\mathrm{Q}$ factor of the first four corpus modes did not show any noticeable difference among different perceptual classes.

\subsubsection{Averaged admittance and radiativity}

To obtain a more clear comparison of different categories in the statistical range (i.e. at higher frequencies), the admittance and radiativity were smoothed in the frequency domain using a $500 \mathrm{~Hz}$ sliding rectangular window and then averaged for different categories (see Fig. 10a and Fig. 10b). The proper way to calculate radiativity would have been to measure the averaged sound pressure over an array of microphones around the instruments. That said, our results using only a single microphone provide a reasonable approximation because they were smoothed in 

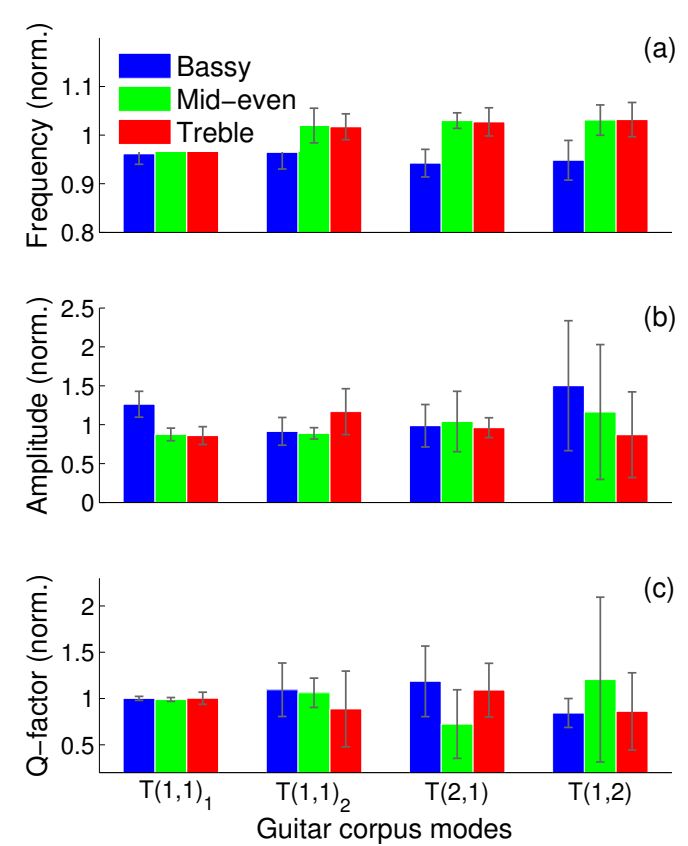

Figure 9: Normalized average frequency (a), amplitude (b), and Q factor (c) for the first four corpus modes for bassy guitars (dark gray bars, blue in colored version), mid-even guitars (light gray bars, green in colored version), and treble guitars (medium gray bras, red in colored version). The error bars show one standard deviation on each side. 
the frequency domain and averaged over different instruments. Since we have already found distinctive features for the instruments of the bassy class, in the subsequent figures the plus/minus standard deviation is eliminated for the bassy class, to help clarity.

It can be seen in Fig. 10a that the band-averaged admittance of the mid-even instruments is greater than or equal to that of the treble instruments over a wide range of frequencies particularly in the range $600 \mathrm{~Hz}$ to $2 \mathrm{kHz}$. Although this dominance does not appear significant for most of the frequencies (i.e., the average for categories overlap when plotted with plus/minus one standard deviation), the mean level of admittance from $600 \mathrm{~Hz}$ to $2 \mathrm{kHz}$ can distinguish mid-even from treble instruments, at least within the pool of our 18 instruments. This result will be further discussed in Sec. 3.5. The band-averaged admittance of the bassy instruments falls between the two other categories over most of the frequencies.

Band-averaged radiativity does not reveal a significant difference among different categories, except a lower average for the bassy guitars in the range from $2 \mathrm{kHz}$ to $2.75 \mathrm{kHz}$ (see Fig. 10b). However, this difference is likely not important as the standard deviation of the bassy instruments in this frequency range was found to be much higher than the other two categories.

\subsubsection{Damping trend}

A method based on time-frequency analysis, first introduced in [17] and most recently applied in [9], was used here to find the trend of the modal damping up to the frequency of $6 \mathrm{kHz}$. As mentioned earlier, the admittances are not compensated for added damping from strings. Fig. 11 shows the averaged damping trend taken over the three perceptual categories.

The $\mathrm{Q}$ factor for all classes rises more sharply from $\mathrm{Q} \approx 30$ at $100 \mathrm{~Hz}$ to $\mathrm{Q} \approx 60$ at $600 \mathrm{~Hz}$ where it reaches a plateau and rises more gradually afterwards. However, the general behavior is not far from linear on a log-log scale. As it can be seen in Fig. 11a and 11b, the value of Q factor over different frequencies and its trend is similar for treble and mid-even classes, which make them indistinguishable by looking at the damping trend. From the power-law fit results, the average power for bassy category is 0.08 and for both categories of mid-even and treble is 0.16 . This results in an average-over-all-instruments of 0.13 , compared to 0.26 and -0.09 reported earlier for the violins and the classic guitars respectively in [9]. Among the instruments of the pool, only one showed a negative power fit. 

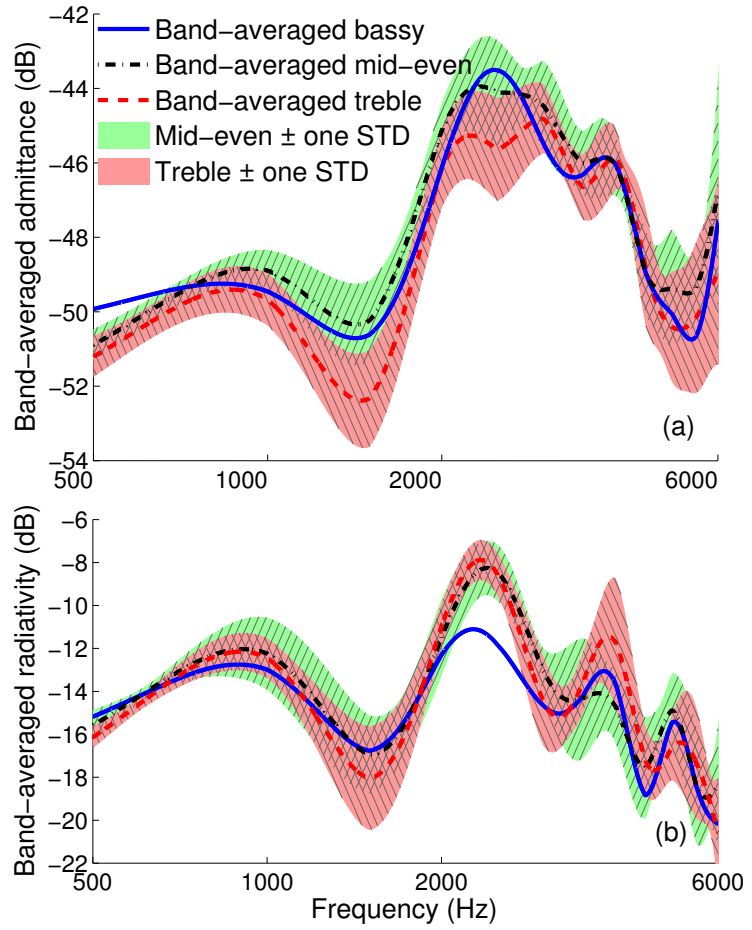

Figure 10: Averaged admittance (a) and radiativity (b) taken over bassy instruments (solid), mid-even instruments (dash-dot) plus/minus one standard deviation (light gray shade, green in colored version), and treble instruments (dashed) plus/minus one standard deviation (darker gray shade, red in colored version). The $\mathrm{dB}$ reference is $1 \mathrm{~m} / \mathrm{sN}$. 

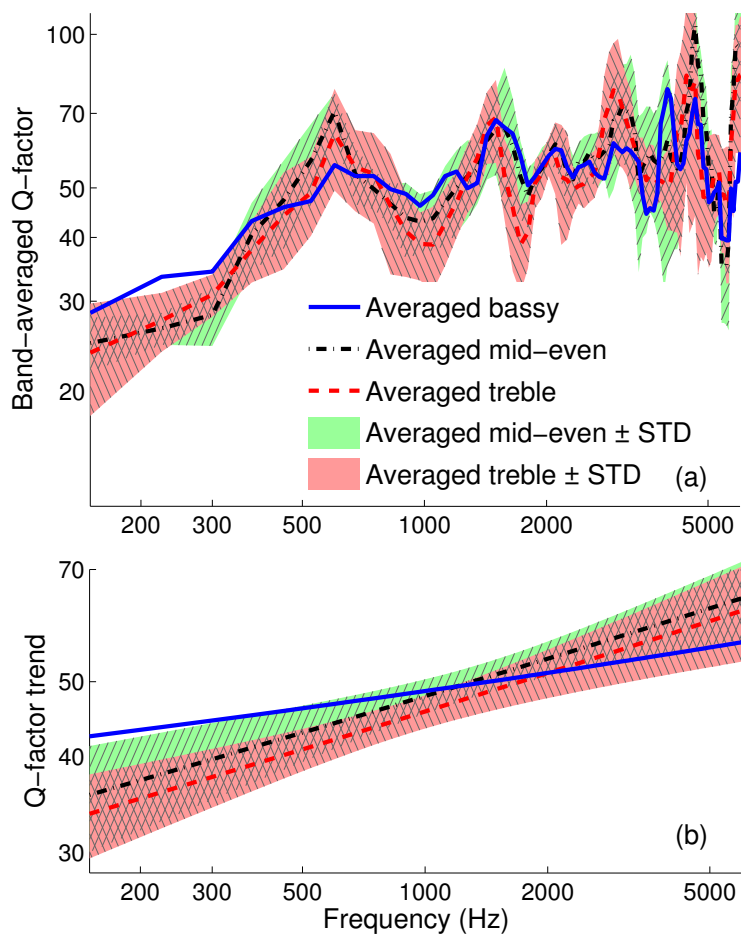

Figure 11: Effective Q-factors averaged for the guitars of different categories plus/minus one standard deviation (solid line for bassy, dash-dot line and light gray / green shade for mid-even, and dashed line and darker gray / red shade for treble), band-average (a) and regression line for power-law behavior (b). Note that vertical axes are different. 


\subsubsection{Harmonic features}

Plucking a guitar string excites multiple harmonics simultaneously which makes it possible that a weighted sum of the admittance over some range of harmonics, rather than the admittance at the fundamental only, is a better way of distinguishing categories of instruments. Forgetting about losses for the moment, the amplitude of the transverse force that the $n$th harmonic of a plucked string applies to the bridge can be found from [5]:

$$
F_{n}=\frac{2 d T_{0}}{n \pi L_{0}} \frac{\beta}{1-\beta} \sin (\beta n \pi)
$$

where $L_{0}$ is the length of the string, $T_{0}$ is its tension, $d$ is the displacement at the plucking point, and $\beta$ is the relative distance of the plucking point from the bridge. This formula does not take into account the possible strong coupling of the plucked string to the body and to other strings, which should be a reasonable approximation for the intended purpose and for most of the notes played on a guitar [16]. The magnitude of the power transfer from the $n$th harmonic of the string to the body is then equal to the bridge velocity multiplied by the applied force, or equivalently

$$
P_{n}=F_{n} v_{n}=F_{n}^{2} G_{\omega}=A\left(\frac{\sin (\beta n \pi)}{n}\right)^{2} G_{\omega}
$$

in which $G_{\omega}$ is the real part of the bridge admittance (also called conductance) and $A$ is a constant assuming a particular string is being played with a constant plucking strength and at a fixed relative distance from the bridge, and again neglecting the losses. This power transfer to the body is largely responsible for the damping of the string mode in question, as well as the radiated sound at that frequency. Based on this idea, two features are calculated for all semitones played on a guitar (from E2 to E6) and averaged over the three perceptual categories. For both cases $\beta$ is arbitrarily chosen as 0.3 , which is a reasonable number for a normal playing condition.

Figure 12a shows the sum of the transferred power by the first 5 harmonics of each played note, here called "power transfer to the body." The choice of 5 harmonics is an arbitrary limit for which the individual harmonics of the string could be perceived individually, and within which most of the energy transfer to the body happens in a normal playing condition. The frequency range that is considered in this fashion would be up to 5 times the highest note 
studied (i.e. $\mathrm{E}_{6}$ ) or equivalently $6.6 \mathrm{kHz}$; this is about the limit up to which the measured admittances show a reasonably good coherence. The unknown constant A in equation (2) could only cause an offset in the results, therefore it is considered to be 1 for convenience. The energy transfer to the body represents the strength of the string/body coupling for a guitar. A large value for this number would result in a strong and more percussive (banjo-like) sound, and a small value would result in a weaker sound with a longer decay (more similar to an electric guitar without an amplifier).

Figure $12 \mathrm{~b}$ shows a slightly different use of the transferred energy by each harmonic. It is calculated by dividing the sum of the energy transferred by the 2nd to 5th harmonic to the energy transferred by the fundamental alone. As well as specifying the ratio of higher harmonic energy to that at the fundamental, this feature defines how the sound evolves once the instrument is plucked. Extremely large or small values for a particular note mean that the note changes its character when it decays, while an average value may be correlated to a more stable sound.

Neither graph shows any clear distinction between the mid-even and treble categories. The bassy category does, however, stand out: most of the patterns in both graphs are shifted downward by a few semitones.

\subsubsection{LTAS and radiativity}

The LTAS results averaged over different categories are illustrated in Fig. 13a. The results for the mid-even instruments are greater than the other two groups over almost all frequencies. Figure 13b is the same as Fig. 13a but the LTAS for each individual instrument is normalized by its area before further processing. After normalization all categories behave similarly over different frequencies meaning mid-even instruments were simply louder than the other instruments, rather than being stronger in a particular range of frequencies.

The band-averaged admittance of the mid-even instruments, earlier presented in Fig. 10, was slightly greater than the treble instruments in some frequency ranges; however, the significance of the stronger LTAS of mid-even instruments, especially beyond $3 \mathrm{kHz}$, can hardly be justified by that alone. A possible explanation is that the mid-even instruments were played systematically harder compared to the treble instruments. There are at least two reasons as to why this might have been the case. First, the guitars were recorded per category starting with the treble and 


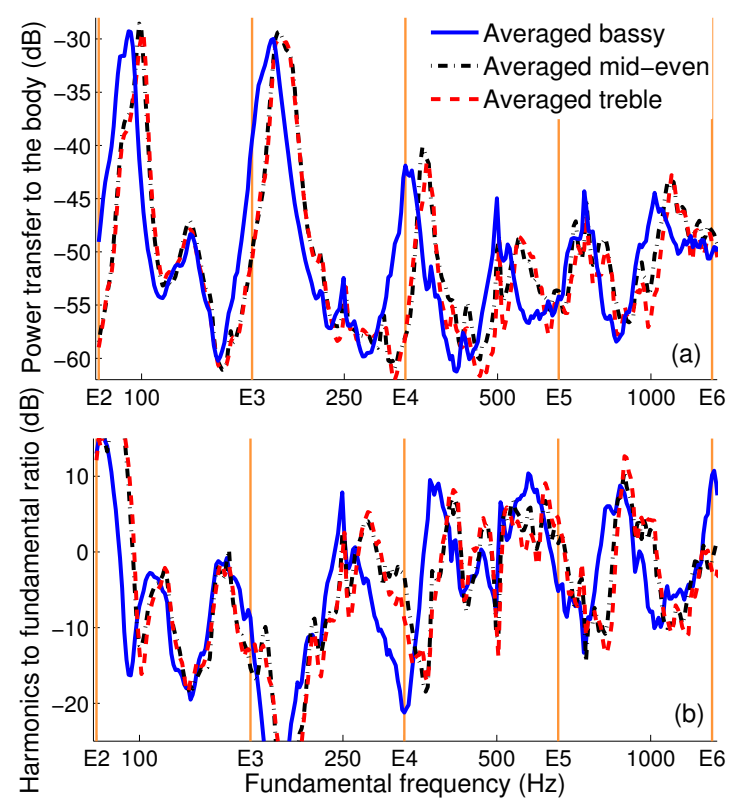

Figure 12: Power transfer to the body (a), and harmonics to fundamental ratio (b) calculated for each played note over the operational range of a guitar $\left(\mathrm{E}_{2}\right.$ to $\left.\mathrm{E}_{6}\right)$, the vertical lines indicate octaves. The values are averaged for the guitars of different categories (solid line for bassy, dash-dot line and light gray / green shade for mid-even, and dashed line and darker gray / red shade for treble).

ending with the mid-even ones. It is therefore possible that the musician felt more confident in the course of the recording session. Second, the mid-even guitars had been described by the manufacturer as having a "more stable" sound. Such a sense might have allowed the performer to play those instruments more freely. Nevertheless, we are aware that the frequency distribution obtained using LTAS can be strongly dependent on the nature of the input signal: depending on the sequence played and used as an input, certain frequency bands may be less excited than others, which may attenuate differences in some frequency regions of interest.

\subsection{Classification results}

According to our results, finding a feature that can distinguish bassy instruments is a relatively easy task. As was shown in Fig. 9a, the frequencies of the first 4 modes for the bassy instruments are around $5 \%$ lower than those for the two other classes. The frequency of the first mode can be determined without much ambiguity or even the need for mode extraction. All of the other 3 low-frequency modes have close adjacent modes, making it harder and sometimes impossible to find their correct modal parameters. 

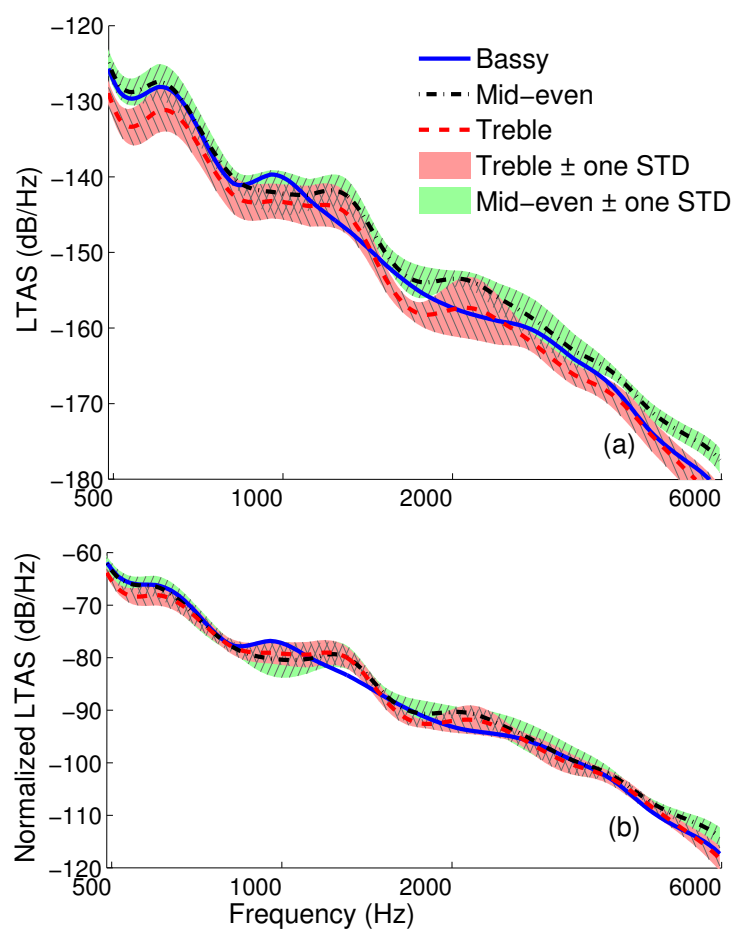

Figure 13: Non-normalized LTAS (a) and normalized LTAS (b) averaged for the guitars of different categories plus/minus one standard deviation (solid line for bassy, dash-dot line and light gray / green shade for mid-even, and dashed line and darker gray / red shade for treble). 
The amplitude of the first mode is another indicator for a bassy instrument that can be used for more assurance (see Fig. 9b). Figure 14a shows a 2-D plane with the normalized frequency and amplitude of the first mode on its horizontal and vertical axes respectively. It can be seen that the bassy instruments are quite distinct from the mid-even and treble ones. Moreover, the guitar with the lowest breathing mode $\left(\mathrm{B} 3, f_{T(1,1)_{1}}=91 \mathrm{~Hz}\right)$ was described by the factory employee as extra-bassy. It should be emphasized that the frequency of the first peak works particularly well as a feature, not because the "bassiness" of the instrument is defined by the notes around this mode's frequency (only the first three notes on the E2 string are below that frequency), but because it represents the overall stiffness-to-weight ratio of the soundbox that effectively scales the frequency of all body modes. It is noteworthy that although $\mathrm{T}(1,1)_{1}$ has a strong Helmholtz component, the amount by which its frequency deviates from the frequency of the pure Helmholtz resonance is strongly dependent on the frequency separation of the pure Helmholtz and pure body breathing modes. The frequency of the pure Helmholtz mode is assumed the same for all instruments of the pool given their very similar geometry (both for the sound box and the sound hole).

The in-plane dimensions of the instruments should have a very small variation percentage given the precise control of geometrical conditions in the factory. Moreover, a lower frequency for the first mode is usually accompanied by a higher amplitude for that mode, which would not normally be the case if such deviation was caused by using a denser wood; this leaves variations in the plate thickness and the equivalent stiffness (i.e., very roughly the geometric mean of the Young's moduli in long-grain and cross-grain directions [14]) as those most likely responsible for the observed shift in mode frequencies. Mode frequencies of a flat plate scale with $\sqrt{E / \rho}\left(t / L^{2}\right)$ where $E$ and $\rho$ are the elastic constant and density, $t$ the thickness and $L$ a characteristic scaling length. If either of the two factors is solely responsible to make a $5 \%$ shift, it would require either a $5 \%$ variation $(\approx 0.125 \mathrm{~mm})$ of the plate thickness or a $10 \%$ variation in the equivalent stiffness. Given the tight tolerances in cutting the plates and their mechanized sanding, it thus appears that variations in equivalent stiffness are the primary reason that some of the instruments in the pool sound more bassy. In the manufacturing process, a wood slab is sliced to produce several plates. The lateral direction of each plate hence represents a particular combination of the slab's radial and tangential directions (due to the growth rings of the tree). This can introduce a significant variation in the plates' stiffness in the lateral direction, which 
produces variations of the effective stiffness of the plates.

Finding a feature that distinguishes mid-even from treble instruments is less obvious. We found that the averaged admittances of the treble instruments in this study are slightly lower than the mid-even instruments in the range of $600 \mathrm{~Hz}$ to $2 \mathrm{kHz}$. Although this difference is not significant over the mentioned range (i.e., average plus/minus one standard deviation of the two categories overlap), it becomes significant if the average level of the admittance for the whole range is compared (i.e., the average value of all frequency points of the admittance from $600 \mathrm{~Hz}$ to $2 \mathrm{kHz}$ ). Figure 14b shows a 2-D plane with the frequency of the first mode on the horizontal axis and the average admittance for the range of $600 \mathrm{~Hz}$ to $2 \mathrm{kHz}$. The difference for the mean of the mid-even and treble categories is about $2 \mathrm{~dB}$. It seems likely that the weaker admittance of the treble category at higher frequencies would contribute to a longer decay for the higher partials. This may contribute to a sharper after-sound of the treble instruments that is known to allow them to cut through the band more effectively. This hypothesis could be tested by recording the plucked sound of the instruments and comparing the decay rate of the partials among different categories. Due to the limited project time frame, this was not investigated further. The mid-even guitars also show stronger LTAS than the treble instruments, though this result might have been due to playing conditions (see Sec. 3.4.5).

We applied the same classification (frequency of $\mathrm{T}(1,1)_{1}$ versus average mobility between $600 \mathrm{~Hz}$ and $2 \mathrm{kHz}$ ) to 6 acoustic guitars from a different model by the same manufacturer (Simon \& Patrick). Unlike the Seagull guitars, which were all nominally identical, some of the S\&P instruments had a slightly different bracing pattern, designed to exemplify the properties of a particular perceptual category. The manufacturer's classification of the 6 guitars was not provided to us prior to our own measurements, though we were told that there were 2 guitars of each category. The resulting classification based on the discussed features is depicted in Fig. 15. The two guitars designed to be more bassy were successfully identified from the frequency of their breathing mode, but distinguishing between the mid-even and treble instruments from the averaged admittance criterion was inconclusive. 

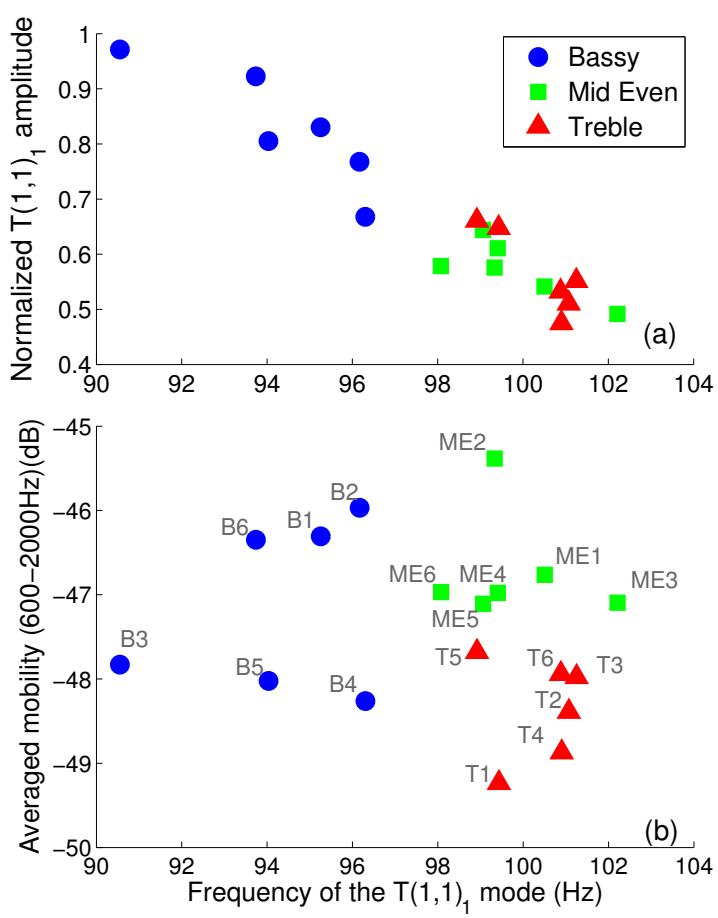

Figure 14: Classification of the acoustic guitars (a) by the frequency and amplitude of their first corpus mode $\mathrm{T}(1,1)_{1}$, only to distinguish bassy instruments from the others; and (b) by the frequency of their first corpus mode $\mathrm{T}(1,1)_{1}$ and their averaged mobility from $600 \mathrm{~Hz}$ to 2 $\mathrm{kHz}(\mathrm{B}=$ bassy, $\mathrm{ME}=$ mid-even, $\mathrm{T}=$ treble $)$. The $\mathrm{dB}$ reference is $1 \mathrm{~m} / \mathrm{sN}$.

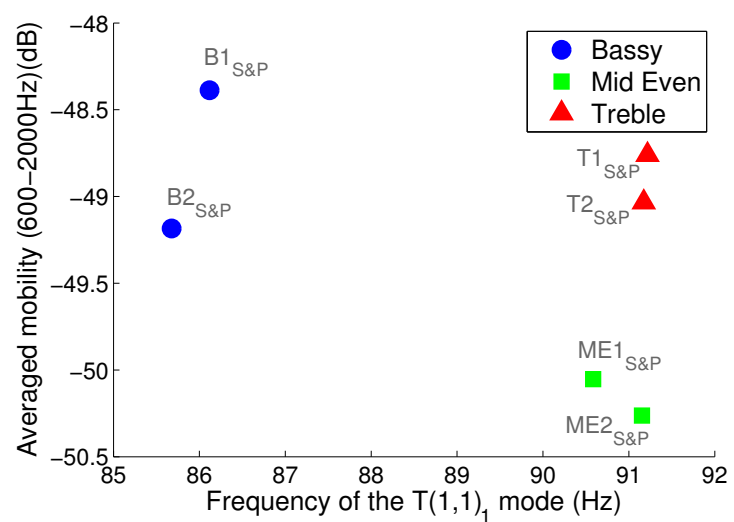

Figure 15: Classification of the six S\&P guitars based on the frequency of the $\mathrm{T}(1,1)_{1}$ mode versus averaged mobility from $600 \mathrm{~Hz}$ to $2 \mathrm{kHz}(\mathrm{B}=$ bassy, $\mathrm{ME}=$ mid-even, $\mathrm{T}=$ treble $)$. The $\mathrm{dB}$ reference is $1 \mathrm{~m} / \mathrm{sN}$. 


\section{Perceptual evaluation}

As previously discussed in Sec. 1, the investigated acoustic guitars were post-classified by the manufacturer based on their experience of what type of instrument might better fit a certain playing style - for example, an amateur musician that plays mostly strummed chords may prefer a bassy guitar, while a more experienced guitarist may opt for a treble instrument. We therefore conducted a perceptual experiment to investigate the level of agreement among guitar players of varying experience and musical background in classifying the guitars into the categories considered by the manufacturer.

\subsection{Method}

The experiment took place in an acoustically dry room with dimensions of $6.0 \times 7.8 \times 3.2 \mathrm{~m}$ and a reverberation time of about $0.3 \mathrm{~s}$ to help minimize the effects of room reflections on the direct sound from the guitars. To avoid the potential problems of using a common pick across all participants (e.g., musicians being uncomfortable with a pick they are not familiar with), participants were asked to carry out the classification task using their own pick(s).

\subsubsection{Participants}

Thirteen guitar players took part in the experiment ( 1 female, 12 males; average age $=25$ yrs, $\mathrm{SD}=5 \mathrm{yrs}$, range $=19-36 \mathrm{yrs}$ ). They had at least 6 years of acoustic guitar experience (average years of training $=11 \mathrm{yrs}, \mathrm{SD}=4 \mathrm{yrs}$, range $=6-20 \mathrm{yrs}$; average hours of practice per week $=14 \mathrm{hrs}, \mathrm{SD}=10 \mathrm{hrs}$, range $=3-40 \mathrm{hrs}$ ) as well as varying experience with different types of guitars [nylon-string (85\%), acoustic-electric (62\%), acoustic bass (15\%), electric (100\%), and electric bass (54\%)]. Participants reported playing a wide range of musical styles [classical (77\%), jazz (85\%), pop-rock (92\%), country / folk / bluegrass (66\%), hard rock / heavy metal (46\%), flamenco (15\%), latin (46\%), blues (77\%), avant-garde / contemporary (54\%), and funk / alternative (46\%)] both in a band and as soloists. Seven guitarists (54\%) reported that they typically or most often use a pick whereas 4 players (31\%) reported they do not; 2 guitarists (15\%) reported using a pick occasionally rather than typically. Nine participants had higherlevel degrees in music performance or other music-related areas [BMus (music theory, classical guitar), BFA (jazz studies), MMus (guitar performance), MA (digital musics), PhD (music 
theory), Diplôme d'État de Professeur de Musique (France)]. All guitar players were paid for their participation.

\subsubsection{Procedure}

The experimental session lasted 2 hours and was organized in two phases. In the first phase, participants were presented with the guitars randomly ordered on the floor (determined by computer calculations). They were asked to play all instruments for up to 20 minutes in order to familiarize themselves with the set and use this time to tune the guitars as needed. In the second phase, participants were given up to 20 minutes to classify each of the guitars as either bassy, mid-even or treble by placing them in specially marked areas of the room. At the end of the second phase, participants were given the opportunity to revise their initial judgements if they so wanted, until they were satisfied with their classification. No playing constraint was imposed on the evaluation process (e.g., specific repertoire or technique). Participants were instead encouraged to follow their own strategy, with the suggestion to use both strummingoriented songs (e.g., Sitting, Waiting, Wishing by Jack Johnson) and fingerpicking-oriented pieces (e.g., Dust in the Wind by Kansas). Participants were instructed to maximize evaluation speed and accuracy. Upon completing the session, participants talked about their classifications and overall experience through written responses to the following questions: "For each class of guitar, what sound characteristics did you look for? Can you describe how you evaluated the guitars in terms of these characteristics (e.g., musical material, technique, style)?" (Q1); "To what extend do you agree with the given classification of guitars?" (Q2); and "Can you suggest a classification that better reflects how you evaluate the sound characteristics of guitars?" (Q3).

\subsection{Results}

Inter-individual consistency was measured as the Fleiss' kappa coefficient between guitar classifications from different participants using a Bayesian approach to inference [18]. Fleiss' kappa is a generalization of Cohen's kappa statistic $\kappa$, a chance-corrected measure of agreement between two assessors, to multiple raters [19]. It ranges from -1 to $1: \kappa(A, B)=1$ and -1 if $A=B$ and $A=-B$, respectively, and $\kappa(A, B)=0$ in case of no association between $A$ and $B$ (i.e., exactly what would be expected by chance). We implemented Gibbs sampling through the software WinBUGS (Windows Bayesian inference Using Gibbs Sampling [20]) with 50,000 observations 


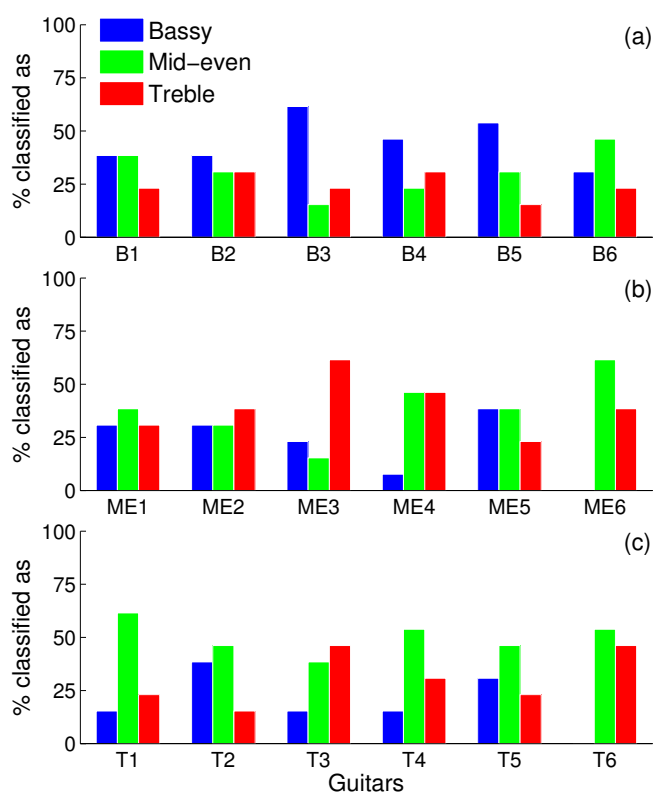

Figure 16: Perceptual evaluation: Proportion of times a guitar was classified into one of the categories by the participants. Guitars are labelled and grouped according to the factory classification $(\mathrm{B}=$ bassy, $\mathrm{ME}=$ mid-even, $\mathrm{T}=$ treble $)$. The color scheme corresponds to the classifications provided by the guitar players.

generated from the posterior distribution, with a burn-in of 1000 observations. A uniformly distributed multicategorical prior was assumed, with total information across all possible cells (i.e., from different combinations of participants) being equivalent to a single prior observation (i.e., a prior sample size equivalent of 1 ).

Fleiss's kappa was estimated with a posterior mean of .01 and a $95 \%$ credible interval [-.02, $.05]$, indicating a very low amount of agreement between the participants in their classifications. Figure 16 shows the proportion of times a guitar was classified as either bassy, mid-even, or treble (the guitars are arranged in three groups according to the initial factory classification). Whereas some agreement concerning the bassy guitars can be observed-indeed, the extra bassy guitar (B3) was most often classified as bassy - the perceptions for the other two classes largely vary across different musicians. In particular, many guitars that had been characterized as treble by the factory employee were perceived as mid-even. Several musicians specifically reported encountering difficulties with the mid-even category. In answering Q3, a player observed that "there is a difference [between] a guitar with dominant mid tones and one with an even sound."

Overall, participants expressed no strong difficulties in carrying out the classification task. 
On a scale from 1 (strongly disagree) to 5 (strongly agree) all performers selected 4 when asked "To what extend do you agree with the given classification of guitars?" This indicates that the 3 categories considered by the manufacturer are generally meaningful to musicians. However, verbal responses to questions Q1 and Q3 reveal that participants often associated bassy with more prominent $\mathrm{E}_{2}$ and $\mathrm{A}_{2}$ strings (i.e., they focused on the low-frequency end of the guitar) and, similarly, treble with better emphasized $\mathrm{B}_{3}$ and $\mathrm{E}_{4}$ strings (i.e., they paid attention to the high-frequency range). As one guitar player noted, "I feel that descriptions like warm, neutral and bright would be easier to classify due to the nature of the instrument. Bass leads my mind to bass strings and to head the low end instead of the entire range from the highest to lowest notes. Same for treble vs bright." In particular, 4 participants used the word "twang" to describe guitars they classified as treble, while another guitarist reported that a treble guitar "sounds best for finger picking and light strumming as heavy playing can get quite ear-piercing and harsh." This last comment seems to echo the second hypothesis for the origin of the stronger LTAS shown by the mid-even guitars (see Sec. 3.4.5).

Two participants reported looking out for problematic notes as a more meaningful way of finding bassy guitars. This likely points toward the "veering" phenomenon associated with a stronger admittance at lower frequencies [16]. Further, one musician referred to the tactile feedback received from a vibrating body, associating bassy guitars with more felt vibrations than the other two categories. This is a perspective shared by the manufacturer as well. In fact, body vibrations can provide tactile and proprioceptive cues that contribute to the perception of the radiated sound, so that the musician can assess their interaction with the instrument cross-modally [21, 22].

\section{Discussion}

A set of 18 steel string guitars of the same model, manufactured with high geometrical tolerances in a modern factory, were evaluated in this study. These guitars were considered by the manufacturer to possess subtle, yet perceivable, variations that could be classified as either bassy, mid-even or treble. The results of this study showed that the instruments classified as bassy had a consistently lower $\mathrm{T}(1,1)_{1}$ breathing mode (see Fig. 9a, 14b and 15). This suggests a lower stiffness-to-weight ratio for the bodies of these guitars, which is most likely the result 
of variations in equivalent stiffness (see Sec. 3.5).

It was further shown that whenever the frequency of $\mathrm{T}(1,1)_{1}$ was shifted downward or upward for a particular guitar, the frequencies of its higher modes consistently changed in a similar way (see Fig. 9; also the slightly shifted bumps at around 600, 750 and $1200 \mathrm{~Hz}$ in Fig. 12a). This would not necessarily be the case if the guitars were of different models (i.e., with varying geometries) or of different types of wood (e.g., plywood versus spruce). This is an important result as there is a practical advantage to having higher-frequency modes move according to the first corpus mode across guitars of the same model. $\mathrm{T}(1,1)_{1}$ is a low-frequency resonance that is sufficiently separated from the adjacent modes and hence easily identifiable. Measuring its frequency across different instruments is easy and affordable in a small workshop environment. One can simply hit the guitar with a regular hammer and record/analyse the sound using an inexpensive microphone and a laptop, reminiscent of the traditional tap-tone method used by luthiers. Moreover, such measurements can be very reliable provided that the boundary conditions of the instrument (i.e., the installed fixture that holds the instrument) as well as the air temperature, pressure and humidity are kept consistent. These parameters constitute the biggest source of uncertainty in frequency-based features, and can easily be controlled in a factory space or small workshop.

Finding a reliable differentiator between the mid-even and treble guitars was less obvious. Although we observed that the averaged mobility curves of the mid-even instruments tended to be slightly higher in the vicinity of $600-2000 \mathrm{~Hz}$ than the treble guitars, further measurements would have been necessary to determine whether the weaker admittance of the treble instruments at higher frequencies would contribute to a longer decay for the higher partials (see Sec. 3.5). In addition, the difference in averaged mobility magnitude between the two categories was only about $2 \mathrm{~dB}$, which is close to the margin of error for the measurement system. Such a small difference in an amplitude-based feature may be easily obscured by variations in the exact angle of the hammer, location of hitting and measuring, or calibration of the sensors.

We invited guitar players of varying musical background and experience to classify the guitars into these categories. We observed very poor agreement among the participants, but it is common in recent perceptual studies of musical instruments that musicians do not agree with each other $[23,24,25]$. This seems a result of them listening to (or feeling) different aspects of the instrument and not necessarily all attending to the same features. The employee 
that post-classified the guitars at the factory was the person that tests every guitar coming off the production line, thus he must have a lot of experience evaluating the sound of these instruments. Assuming he did his classification carefully, then there likely were particular things he was listening for. The participants in our perceptual experiment would not necessarily listen for the same features, so it is not too surprising they largely disagreed on their classifications. That said, the differences between the treble and mid-even guitars were extremely subtle and even company representatives often mis-categorized them during informal playing tests. And this, together with the fact that our measurements showed no consistent differences between the two categories, would seem to lead us to question whether the distinction between mid-even and treble truly exists at all. Another possible explanation is that different musicians may follow different processes to assess those features considered essential for the evaluation of an instrument $[23,26]$. Accordingly, guitar players may agree of what particular characteristics they look for in a treble guitar, but the perception of the same characteristics widely varies across individuals.

\section{Conclusions}

Efforts to understand how the tonal quality of an instrument relates to its measurable physical properties have often been inconclusive (e.g., [27]). In the light of some recent studies, the concept of "good" versus "bad" instruments is likely too general and it would be more advantageous to instead consider specific perceptual attributes of an instrument $[23,28]$. In the long run, it may be possible to derive a multidimensional timbre space where each dimension is explained by some measurable quantities, and in which instruments with similar qualities are likely to cluster. In this study, more specific sound characteristics of instruments were thus targeted rather than their overall tonal quality.

From bridge admittance measurements on 18 steel string guitars from the same production line and post-classified by the manufacturer as either bassy, mid-even or treble (see Sec. 1 for details), it was shown that it is possible to discriminate between bassy guitars and the other two categories based on the frequency of the breathing mode. However, finding a similarly reliable feature to distinguish mid-even from treble instruments is less trivial at this point. The treble guitars demonstrated a lower averaged mobility in the frequency range of 600-2000 
$\mathrm{Hz}$, though more empirical investigation would be necessary before drawing any conclusions. Results from a perceptual experiment, wherein guitar players were asked to classify the guitars into these categories, showed almost no agreement between guitarists in their judgements as well as between the latter and the classification by the manufacturer. Despite the overall lack of consensus, guitar players tended to agree more on the bassy instruments than the other two categories.

Some essential additions to this study are a more careful analysis on the perceptual definition of mid-even versus treble, and analysing the decay rate of the partials for a plucked sound to see how it correlates with the admittance. Analysis of this kind has been performed for synthesized acoustic guitar sounds (see Fig. 7 in [16]) but a similar analysis on real guitar sounds will involve difficulties caused by the double set of harmonics [29] as well as longitudinal string vibrations (also dubbed "phantom partials") [30].

\section{Acknowledgements}

This project was funded by a Natural Sciences and Engineering Research Council of Canada Engage grant in collaboration with Godin Guitars. The authors wish to thank Robert Godin for this opportunity. We also thank Lawrence Joseph (statistics) and Garry Antonio (guitar performance) for constructive comments. We are grateful to Jim Woodhouse for sharing his analysis code. The first author would like to acknowledge the Government of Canada for a Vanier Canada Graduate Scholarship.

\section{References}

[1] E. V. Jansson, "A study of acoustical and hologram interferometric measurements of the top plate vibrations of a guitar," Acust. Acta Acust., vol. 25, no. 2, pp. 95-100, 1971.

[2] O. Christensen and B. B. Vistisen, "Simple model for low-frequency guitar function," J. Acoust. Soc. Am., vol. 68, no. 3, pp. 758-766, 1980.

[3] O. Christensen, "Quantitative models for low frequency guitar function," J. Guitar Acoustics, vol. 6, no. 2, pp. 10-25, 1982. 
[4] H. Wright, The acoustics and psychoacoustics of the guitar. PhD thesis, Dept. of Physics and Astronomy, University of Wales, Cardiff, UK, 1996.

[5] N. H. Fletcher and T. D. Rossing, The Physics of Musical Instruments. New York: Springer, second ed., 1998.

[6] J. E. Popp, "Four mass coupled oscillator guitar model," J. Acoust. Soc. Am., vol. 131, no. 1, pp. 829-836, 2012.

[7] J. Woodhouse, "Body vibration of the violin — what can a maker except to control?," Catgut Acoust. Soc. J. (Series II), vol. 4, no. 5, pp. 43-49, 2002.

[8] B. Elie, F. Gautier, and B. David, "Macro parameters describing the mechanical behavior of classical guitars," J. Acoust. Soc. Am., vol. 132, no. 6, pp. 4013-4024, 2012.

[9] J. Woodhouse and R. S. Langley, "Interpreting the input admittance of violins and guitars," Acust. Acta Acust., vol. 98, pp. 611-628, 2012.

[10] A. Gabrielsson and E. V. Jansson, "Long-time-average-spectra and rated qualities of twenty-two violins," Acustica, vol. 42, no. 1, pp. 47-55, 1979.

[11] P. D. Welch, "The use of Fast Fourier Transform for the estimation of power spectra: A method based on time averaging over short, modified periodograms," IEEE Trans. Audio and Electroacoust., vol. AU-15, pp. 70-73, 1967.

[12] J. Sundberg, "The singer's formant revisited," J. Voice, vol. 4, pp. 106-119, 1995.

[13] A. Buen, "On timbre parameters and sound levels of recorded old violins," J. Violin Soc. Am.: VSA Papers, vol. XXI, no. 1, pp. 57-68, 2007.

[14] J. Woodhouse, "On the "bridge hill" of the violin," Acust. Acta Acust., vol. 91, pp. 155-165, 2005 .

[15] B. Richardson, "The acoustical development of the guitar," Catgut Acoustical Society Journal, vol. 2, no. 5, pp. 1-10, 1994.

[16] J. Woodhouse, "On the synthesis of guitar plucks," Acust. Acta Acust., vol. 90, pp. 928-944, 2004. 
[17] C. H. Hodges, J. Power, and J. Woodhouse, "The use of the sonogram in structural acoustics and an application to the vibrations of cylindrical shells," J. Sound Vib., vol. 101, pp. 203$218,1985$.

[18] L. D. Broemeling, Bayesian Methods for Measures of Agreement. Biostatistics, New York: Chapman \& Hall/CRC, 2009.

[19] J. L. Fleiss, "Measuring nominal scale agreement among many raters," Psychological Bulletin, vol. 76, no. 5, pp. 378-382, 1971.

[20] D. Lunn, C. Jackson, N. Best, A. Thomas, and D. Spiegelhalter, The BUGS Book: A Practical Introduction to Bayesian Analysis. Texts in Statistical Science, New York: Chapman \& Hall/CRC, 2012.

[21] C. Saitis, Evaluating violin quality: Player reliability and verbalization. PhD thesis, Dept. of Music Research, McGill University, Montreal, Quebec, Canada, 2013.

[22] I. Wollman, Perception bimodale des violonistes en situation de jeu: Influence des retours auditif et vibrotactile sur l'évaluation du violon. $\mathrm{PhD}$ thesis, Université Pierre et Marie Curie, Paris, France, 2013.

[23] C. Saitis, B. L. Giordano, C. Fritz, and G. P. Scavone, "Perceptual evaluation of violins: A quantitative analysis of preference judgements by experienced players," J. Acoust. Soc. Am., vol. 132, no. 6, pp. 4002-4012, 2012.

[24] C. Fritz, J. Curtin, J. Poitevineau, P. Morrel-Samuels, and F.-C. Tao, "Player preferences among new and old violins," Proc. Nat. Acad. Sci. USA, vol. 109, no. 3, pp. 760-763, 2012.

[25] P. Eveno, J.-P. Dalmont, R. Caussé, and G. P. Scavone, "An acoustic and perceptual evaluation of saxophone pad "resonators"," Acust. Acta Acust., Submitted.

[26] C. Fritz, J. Woodhouse, F. P.-H. Cheng, I. Cross, A. F. Blackwell, and B. C. J. Moore, "Perceptual studies of violin body damping and vibrato," J. Acoust. Soc. Am., vol. 127, no. 1 , pp. 513-524, 2010.

[27] G. Bissinger, "Structural acoustics of good and bad violins," J. Acoust. Soc. Am., vol. 124, no. 3, pp. 1764-1773, 2008. 
[28] C. Saitis, C. Fritz, C. Guastavino, and G. P. Scavone, "Conceptualization of violin quality by experienced performers," in Proc. Stockholm Music Acoust. Conf., (Stockholm, Sweden), pp. 123-128, 2013.

[29] J. Woodhouse, "Plucked guitar transients: Comparison of measurements and synthesis," Acust. Acta Acust., vol. 90, pp. 945-965, 2004.

[30] B. Bank and L. Sujbert, "Generation of longitudinal vibrations in piano strings: From physics to sound synthesis," J. Acoust. Soc. Am., vol. 117, no. 4, pp. 2268-2278, 2004. 